\title{
The Multiple Roles of sGP in Ebola Pathogenesis
}

\author{
Marc-Antoine de La Vega,, ${ }^{1,2}$ Gary Wong, ${ }^{1,3}$ Gary P. Kobinger, ${ }^{1-4}$ and Xiangguo Qiu ${ }^{1}$
}

\begin{abstract}
Ebola causes severe hemorrhagic fever in humans and nonhuman primates, and there are currently no approved therapeutic countermeasures. The virulence of Ebola virus (EBOV) may be partially attributed to the secreted glycoprotein (sGP), which is the main product transcribed from its GP gene. sGP is secreted from infected cells and can be readily detected in the serum of EBOV-infected hosts. This review summarizes the multiple roles that sGP may play during infection and highlights the implications for the future design of vaccines and treatments.
\end{abstract}

\section{Introduction}

$\mathbf{E}$ BOLA IS AN ENVELOPED negative-stranded RNA virus. It belongs to the family Filoviridae, which is composed of three genera: Ebolavirus, Marburgvirus, and Cuevavirus. There are currently five species of Ebolavirus: Reston ebolavirus, Taï Forest ebolavirus, Sudan ebolavirus, Zaire ebolavirus, and Bundibugyo ebolavirus, which contain one virus each: Reston virus (RESTV), Taï Forest virus (TAFV), Sudan virus (SUDV), Ebola virus (EBOV), and Bundibugyo virus (BDBV), respectively. The Marburgvirus genus consists of a single species, Marburg marburgvirus, which is divided into Marburg virus (MARV) and Ravn virus (RAVV) (29). EBOV is the etiological agent of Ebola virus disease (EVD) in humans and nonhuman primates. EVD has a short incubation time ranging from 2 to 21 days, and initial signs of disease are characterized by the onset of flu-like symptoms, such as fever, myalgia, and a general malaise, before rapidly progressing toward hemorrhage, shock, and multiorgan failure (16). Death is the outcome in up to $90 \%$ of human cases. Lloviu virus (LLOV), which belongs to the new genus Cuevavirus, was recently discovered in insectivorous bats in a Spanish cave. However, its pathogenicity in humans is unknown because no human cases were ever reported. Furthermore, infectious viruses could not be isolated from these bats $(32,41)$.

EBOV has drawn considerable attention since its discovery in 1976 due to sporadic but devastating human outbreaks in Africa, as well as its suitability as a biological weapon. EBOV is classified as a Category A Priority pathogen by NIAID and a Category A Agent of Bioterrorism by the CDC. There are currently no prophylactic or therapeutic protocols approved for human use, and current treatments consist only of palliative care (6). This contributes to the many reasons why this virus is so feared, and why there is a need to develop a protective vaccine or curative treatment. Currently, more studies are needed to understand Ebola pathogenesis, with the view to develop more effective vaccines and antivirals in the future. This review will focus on the secreted glycoprotein (sGP), a viral protein produced during infection by the members of the genus Ebolavirus. The currently available research will be discussed in-depth, including the structure of sGP, its multifunctional roles during EBOV infection, and in particular, its immunomodulatory capabilities.

\section{Virological and Structural Studies of SGP}

sGP is encoded by the GP gene of all five species of Ebolavirus. It is initially synthesized as pre-sGP, a Golgispecific precursor (52), which undergoes post-translational proteolytic cleavage at its C-terminus by cellular proteases, such as furin (55), to yield the mature form of the protein. Furin cleaves pre-sGP at the amino acid position 324 at the $\mathrm{R}-\mathrm{X}-\mathrm{R}-\mathrm{R}$ site, which is conserved among the different subtypes of EBOV (52). The cleavage product formed at the $\mathrm{C}$-terminus, $\Delta$-peptide, is a peptide that is highly modified at the post-translational level. The $\Delta$-peptide is retained for a longer period in producer cells compared to sGP, before being released into the extracellular medium (52). A recent study showed that this peptide inhibits the entry of Ebola into target cells by binding to receptors on filovirus-permissive cells (44). However, the receptors are still unknown (38), and the target of the $\Delta$-peptide remains a mystery. Interestingly, the $\Delta$-peptide also prevented the MARV entry, which suggests that it affects a general mechanism for filovirus entry (44), considering that MARV does not produce sGP or $\Delta$-peptide.

\footnotetext{
${ }^{1}$ Special Pathogens Program, National Microbiology Laboratory, Public Health Agency of Canada, Winnipeg, Manitoba, Canada. Departments of ${ }^{2}$ Immunology and ${ }^{3}$ Medical Microbiology, University of Manitoba, Winnipeg, Manitoba, Canada.

${ }^{4}$ Department of Pathology and Laboratory Medicine, University of Pennsylvania School of Medicine, Philadelphia, Pennsylvania.
} 
Subsequent to proteolytic cleavage, sGP monomers bind to each other in a parallel orientation and are linked by two disulfide bonds, at residues Cys53 and Cys306 to form a $110 \mathrm{kDa}$ homodimer $(5,15,51)$. It was also predicted that sGP contains six $N$-linked glycosylation sites, but only five were shown to be used consistently (15). The effects of these posttranslational modifications are unknown regarding pathogenesis, but certainly, their roles would be interesting to define. EBOV sGP was also the first protein among any viruses shown to be C-mannosylated. In this particular case, C-mannosylation was detected on Trp288 (14), which is part of the C-mannosylation recognition motif W-X-X-W (18).

The various EBOV glycoproteins are coded by the $G P$ gene through transcriptional editing. The fourth gene of the EBOV genome, GP, encodes three proteins of different sizes: the full-length 676-residue surface glycoprotein $\left(\mathrm{GP}_{1,2}\right)$, the 364-residue presecreted glycoprotein (pre-sGP) (46), and the 298-residue small secreted glycoprotein (ssGP) $(51,53) . \mathrm{GP}_{1,2}$ is produced as a result of a slippage or stuttering by the viral polymerase at the editing site (seven consecutive template uridines) in the $G P$ gene $(36,46,53)$. This adds an eighth adenosine residue to the transcribed mRNA, overriding the premature end to $G P$ transcription, and allowing for the fusion of the two separate open reading frames (ORFs), which results in the mRNA encoding $\mathrm{GP}_{1,2}$. The unedited mRNA transcripts lead to the production of sGP, whereas the addition of two additional adenosine residues during this process (resulting in nine adenosines) leads to the production of ssGP, a secreted N-glycosylated homodimer, in which the function still remains unclear (36). This mechanism, called transcriptional editing, was first described in viruses of the Paramyxoviridae family concerning their $P$ gene $(22,28,30)$.

The latest available experimental data for the GP gene show that the transcripts coding for these proteins (sGP: $\mathrm{GP}_{1.2}: \mathrm{ssGP}$ ) are produced at a ratio of $71: 24: 5$ in Vero E6 cells (36), confirming previous data showing that sGP is the main product resulting from GP transcription (43). However, this ratio has never been confirmed at the protein level, in either in vitro or in vivo studies. The consequences of transcriptional editing were explored in a recombinant EBOV with a deletion of the editing site, in which the recombinant virus could not produce sGP. A significant increase in cytopathogenicity was observed during in vitro infections with the recombinant virus compared to the wild-type (WT) EBOV virus $(1,56)$. Interestingly, in vivo studies have shown the opposite where exclusive production of GP was associated with reduced pathogenicity and ultimately reversion to the WT editing site. Reversion resulted in a return to normal expression levels of sGP and restoration of the highly pathogenic phenotype (50). This shows the importance of RNA editing in regulating $\mathrm{GP}_{1,2}$ expression. Increasing cytotoxicity during EBOV infection by promoting the production of sGP at the expense of $\mathrm{GP}_{1,2}$ may result in a more favorable environment for virus replication in vivo.

Although sGP is the main product of $G P$, its role in Ebola pathogenesis is hypothetically diverse but often speculative because the majority of investigations into sGP have been focused on structural studies. sGP is a nonstructural protein, which shares its first 295 residues with $\mathrm{GP}_{1,2}(46,47,53)$. In 2011, a study described for the first time a potential structural role for sGP (25). Indeed, in vitro analyses showed that
sGP was likely able to bind $\mathrm{GP}_{2}$ and act as a substitute for $\mathrm{GP}_{1}$ since Human Embryonic Kidney 293T (HEK293T) cells coexpressing sGP or pre-sGP and $\mathrm{GP}_{2}$ were able to bind to KZ52 (25). KZ52 is a monoclonal antibody isolated from a human survivor during the 1995 EBOV outbreak in Kikwit, and its binding site was shown to be at the interface between $\mathrm{GP}_{1}$ and $\mathrm{GP}_{2}$ (31). This sheds light on a potentially novel role of sGP as a structural protein. Cells that expressed sGP, pre-sGP, or $\mathrm{GP}_{2}$ only were unable to bind to KZ52, arguing in favor of an interaction between the two proteins. The same study also showed that this complex was able to mediate infection in a vesicular stomatitis virus $\Delta$ glycoprotein pseudotyped model (25). It was previously shown that $\mathrm{GP}_{1,2}$ was responsible for, or at least part of, the cytotoxicity observed in EBOV-infected cells. Two regions of the surface glycoprotein were identified as having potentially a cytotoxic effect, the extracellular region of $\mathrm{GP}_{2}$ (10) and the mucin-like domain of $\mathrm{GP}_{1}$ (65). This led the authors to hypothesize that the replacement of $\mathrm{GP}_{1}$ by sGP could play a role in reducing cytotoxicity, considering that it is likely that these sGP-GP 2 complexes associate with $\mathrm{GP}_{1,2}$ to form the trimers on the virus surface. However, these initial findings were never substantiated by subsequent studies and therefore additional work will be required to understand better the possible significance of sGP as a structural protein.

\section{SGP and the Pathogenesis of Ebolavirus Infection}

\section{sGP and neutrophils}

It was previously hypothesized that sGP could have different roles to play in affecting aspects of host immunity. An initial study suggested that sGP had the ability to bind to and inactivate neutrophils through CD16b, a neutrophil-specific $\mathrm{Fc} \gamma$ receptor III (64), which is one of the key Fc receptors for recruitment of polymorphonuclear leukocytes following the binding of immune complexes. Further studies showed that this inactivation could be linked to the disruption of Fc $\gamma$ RIIIB with CR3, which is a complement receptor important for the signaling of CD16b (27). However, these findings were rapidly challenged by others because the original study omitted that the observed interaction between sGP and neutrophils could also be mediated by the Fc portion of the anti-sGP antibody binding with Fc $\gamma$ RIIIB (33). It was later shown that the binding observed initially was indeed the result of the formation of an immune complex between CD16b and the Fc portion of the anti-sGP antibody. This was shown through the use of a Fab fragment, which failed to detect sGP binding to neutrophils (48). Together, these results argue against a direct interaction between sGP and neutrophils, and to date, there has not been any new data to support the existence of an interaction between sGP and neutrophils.

\section{sGP and lymphocyte apoptosis}

During an EBOV infection, it is observed that B and $\mathrm{T}$ cells, which are not targeted for infection by the virus, undergo bystander apoptosis by an unknown mechanism (2-4,7,8,1921,58). Since sGP can be detected in large amounts in the bloodstream, it was suggested that sGP could cause this phenomenon (46) by interacting with circulating lymphocytes. 
Previous data also suggest that the observed apoptosis could occur through both the intrinsic and extrinsic pathways $(8,21,23)$. In 2011, Wolf et al. investigated the association between sGP and apoptosis (60). In a first experiment, they assessed the effect of recombinant sGP on the apoptosis of Jurkat cells, a T-lymphocyte cell line. However, cell death was not observed, indicating that sGP was not able to induce apoptosis by itself. The authors then assessed the costimulatory effect of recombinant sGP on death receptor-induced apoptosis because it was reported that lymphocyte apoptosis in human EBOV infection could be mediated through Fas/FasL interactions (58). To this end, they stimulated Jurkat cells with known death receptors ligands, including TRAIL, FasL, or $\mathrm{TNF}-\alpha$ and then used sGP for costimulatory purposes. They found that the presence of sGP did not have any effect on the levels of apoptosis when paired with any of the three death receptor ligands, which indicate that sGP does not modulate apoptosis through the extrinsic pathway.

Further studies are required to investigate the ability of sGP to induce apoptosis through the intrinsic pathway. However, much broader questions may need to be addressed first. A recent report by Olejnik et al. suggests that EBOV does not induce apoptosis in infected cells, including macrophages (42). The authors were not able to detect translocation of phosphatidylserine by annexin $\mathrm{V}$ staining or caspase activation by a technique called fluorochrome inhibitor of caspase (FLICA) staining in EBOV-infected cells, which argues against current evidence showing that EBOVinfected cells undergo apoptosis. To strengthen their point, the authors also studied the state of PARP-1, a substrate to various cellular proteases. Depending on the mechanism involved, the cleavage of PARP-1 yields different fragments that have previously been identified as makers for either apoptosis or necrosis (9). In accordance with their hypothesis, they detected low levels of apoptotic-specific fragments in EBOV-infected cells, whereas higher levels of necroticspecific fragments were detected in the same cells. However, the authors did not focus on necrosis in bystander cells, including $\mathrm{B}$ and $\mathrm{T}$ lymphocytes. Caspase activation was detected by FLICA staining in noninfected cells $48 \mathrm{~h}$ postinfection, in the same way that single noninfected apoptotic cells by annexin V staining was also detected. Whether apoptosis of bystander cells was caused by the production of sGP in the extracellular medium by infected cells still remains to be clarified.

\section{sGP and vascular dysregulation}

It was previously hypothesized that sGP could also be responsible for the endothelial modifications observed during EBOV infection, including vascular dysregulation. To this end, Wahl-Jensen and Afanasieva showed that the two main sGP by-products, sGP and $\Delta$-peptide, were unable to activate endothelial cells (57). This was demonstrated by the absence of upregulation of cellular adhesion molecules (CAMs), such as ICAM-1, VCAM-1, or E-selectin, following the addition of the recombinant forms of secreted EBOV glycoproteins, which include not only sGP but also its $\Delta$-peptide, to endothelial cells. On the other hand, viruslike particles expressing $\mathrm{GP}_{1,2}$ were able to upregulate the previously mentioned CAMs. They further characterized the role of sGP in the pathogenesis of EBOV by looking at its ability to influence the endothelial cell barrier function. Again, sGP did not have a significant impact when added to the cells, as represented by the absence of changes in VEcadherin arrangement, actin organization, or transepithelial electrical resistance when measured by impedance spectroscopy (57). In a subsequent experiment, the authors treated endothelial cells with sGP and TNF- $\alpha$, which caused a decrease in barrier function. It was observed that the addition of sGP was able to partially restore barrier function (57), suggesting an anti-inflammatory role for sGP.

Further studies by the authors led to a better understanding of the relationship between the structure of sGP and its antiinflammatory role. They showed that C-mannosylation on Trp288 was not required for the anti-inflammatory activity of sGP (14), but mutations of Cys53Gly, Cys306Gly, as well as the double mutant all had impaired ability to rescue the endothelial barrier function (15). Therefore, a structurally stable sGP homodimer is necessary to support this function. This led others to investigate whether sGP was able to modulate the interaction of lymphocytes with activated endothelial cells. For this purpose, a coculture model between human umbilical vein endothelial cells (HUVECs) and Jurkat cells was used, in which TNF- $\alpha$-stimulated HUVECs allowed for the adhesion of Jurkat cells, whereas their unstimulated counterparts did not. However, the addition of recombinant sGP did not influence the adhesion of Jurkat cells to HUVECs (60), suggesting that sGP alone has no effect on the adhesion of lymphocytes. Overall, these studies suggest that sGP might not have a key role when it comes to vascular dysregulation during an Ebola infection. However, its influence on barrier function was shown, and further studies are required to demonstrate the extent of this possible new role and the mechanism behind it.

\section{Immune Modulation of Host Responses by sGP}

\section{sGP as a decoy antigen}

Since sGP is the main product of the GP gene and secreted from the Ebola-infected cells, it is not surprising to be able to detect sGP in the blood of infected patients (46) and nonhuman primates. It has been shown in the past that antibodies found in the sera of human EHF survivors crossreacted with sGP rather than $\mathrm{GP}_{1,2}$ (34) and that sGP was able to inhibit the virus-specific neutralizing activity of GP antisera (24). Likewise, it was shown, in a vaccinated mouse model using a Venezuelan equine encephalitis replicon producing both $\mathrm{GP}_{1,2}$ and $\mathrm{sGP}$, that elicited antibodies would cross-react between the two proteins (59). Since $\mathrm{GP}_{1,2}$ and sGP share a common N-terminus, it was hypothesized that sGP could play a role in controlling host humoral immune responses by adsorbing elicited antibodies raised against $\mathrm{GP}_{1,2}$. As mentioned previously, sGP production is favored in vivo, suggesting that sGP is important for virus survival within the host.

\section{sGP and the subversion of the immune system}

Recent studies have established that the production of specific antibodies following Ebola infection was important for host survival and correlated with protection $(35,62)$. The target of choice for antibody production is the surface glycoprotein $\mathrm{GP}_{1,2}$ since it is the only protein available on the 
surface of the virion. Interestingly, it is also the most immunogenic of the EBOV antigens as determined by immunization studies using DNA vaccines expressing independent EBOV antigens $(49,63)$. These experiments established that GP was able to induce a broader immune response, both at the cellular and humoral level, at least compared to the nucleoprotein of EBOV $(49,63)$. However, the virus is also able to counteract these efforts due to the heavy glycosylation of $\mathrm{GP}_{1,2}$, which shield the virus through epitope masking (12) [reviewed by Wong et al. (61)]. Glycosylation of $\mathrm{GP}_{1,2}$ and related immune responses have also been extensively studied in the past. Dowling et al. were the first one to show the impact that glycosylation of $\mathrm{GP}_{2}$ had on antigenicity and immunogenicity of EBOV GP. The same study also suggests that the deletion of the mucin-rich domain negatively influences immunogenicity (13). This heavily glycosylated region was thought to hide critical epitopes for protection as vaccination of mice with GP constructs lacking this domain conferred less protection than other constructs that possessed this mucin-rich domain. However, further investigations are required as this was not expected, given available data on Ebola (12) and other viruses, such as human immunodeficiency virus $(11,26,40,45)$.

In 2012, Mohan et al. (39) demonstrated that sGP was able to compete for binding anti-GP $\mathrm{GP}_{1,2}$ antibodies induced by sGP, but not by $\mathrm{GP}_{1,2}$. They also showed that sGP can interfere with antibody-mediated neutralization, but only in animals that have been previously exposed to sGP (39). This led them to hypothesize that a potential role for sGP could be to redirect the host immune response against epitopes that are either inaccessible or not present in $\mathrm{GP}_{1,2}$, or toward epitopes that are shared between the two proteins. This would then allow sGP to effectively adsorb anti-GP ${ }_{1,2}$ antibodies due to its abundance in the serum relatively to $\mathrm{GP}_{1,2}$. In their proposed model, they consider that the preinfected host starts with a repertoire of naive $\mathrm{B}$ cells that are able to recognize both $\mathrm{GP}_{1,2}$ and sGP. However, because sGP is produced in larger amounts and more dimers are formed from the same number of molecules than GP trimers, B cells recognizing sGP-specific epitopes or epitopes shared between the two isoforms are more likely to encounter sGP. Based on previous work by Mehedi et al. (36), this means that for every 100 monomers produced from the $G P$ gene, $\sim 35$ sGP dimers, 8 GP trimers, and 2 ssGP dimers can be obtained. So while there was a 2.8:1 ratio (sGP:GP) at the transcript level, the same ratio is almost double at the protein level (4.4:1). In addition to the sheer numbers favoring sGP absorbing the majority of GP-specific antibodies, another possibility that could explain this phenomenon is that the number of sGPspecific B cells could also increase and outcompete the B cells that recognize $\mathrm{GP}_{1,2}$-specific antibodies for antigen and survival signals. This would result in a humoral response biased toward epitopes specific to sGP or epitopes shared between $\mathrm{sGP}$ and $\mathrm{GP}_{1,2}$.

This model could also explain why anti-GP ${ }_{1,2}$ antibodies from animals primed with sGP and then boosted with $\mathrm{GP}_{1,2}$ maintain cross-reactivity with sGP. When the animals are first vaccinated with sGP, the naive B cells that expand recognize sGP-specific epitopes and/or epitopes shared between sGP and $\mathrm{GP}_{1,2}$. By the time the animals are boosted with $\mathrm{GP}_{1,2}$, the number of memory $\mathrm{B}$ cells that are able to recognize shared epitopes greatly exceed the number of naive $B$ cells recognizing unshared epitopes. Therefore, the anti-sGP memory B cells can be preferentially activated, resulting in their expansion and boosting the anti-sGP response. This could mean that immunization of a previously infected individual with a vaccine encoding $\mathrm{GP}_{1,2}$ could possibly boost mainly an existing non-protective antibody response.

One of the most interesting findings of this study is the antigenic subversion of the humoral response by sGP. If mice were vaccinated with $\mathrm{GP}_{1,2}$ and then boosted with sGP, it was observed that the anti-GP ${ }_{1,2}$ response was rendered susceptible to sGP competition. One possible explanation is that even if the activated B cells in these animals are mainly directed toward epitopes that are not shared with sGP, a small subset of sGP-reactive B cells would still be present. When the animals are boosted with sGP, these B cells could expand to the detriment of the ones that are able to recognize only $\mathrm{GP}_{1,2}$-specific epitopes. In practice, this would represent a situation where an individual would at first be vaccinated against $\mathrm{GP}_{1,2}$, which is the antigen included in most experimental EBOV vaccines and then be exposed to EBOV. In the event where an individual could not rapidly clear the virus due to an insufficient vaccine-mediated immune response, sGP could build up through viral replication and progressively shift the antibody response toward itself, thereby rendering the immunization ineffective.

\section{Discussion}

The understanding of EBOV pathogenesis is crucial for the development of efficacious treatments and vaccines. Part of the answer may reside in the control of sGP during EBOV infections. According to available data, sGP could be involved at many different levels, as summarized in Figure 1, ranging from restoring barrier functions of endothelial cells to modulating the immune response. However, many other roles that have been suggested for sGP still require more indepth investigations. Currently, there is no conclusive evidence that sGP has any impact on bystander apoptosis of B and T lymphocytes. The effect of sGP as a structural protein in Ebola pathogenesis also requires further investigation because right now we can only speculate about its impact in the potential reduction of cytotoxicity through the replacement of $\mathrm{GP}_{1}$. Finally, one of the most interesting questions that still demands an answer is the mechanism behind sGPmediated immune subversion, skewing the host antibody response toward itself. This may need to be considered for designing novel treatments or vaccine strategies.

It would also be interesting to quantitate the levels of sGP produced by viruses from each species of Ebolavirus and to determine whether it correlates with their respective levels of pathogenicity. For example, the observation that SUDV and BDBV produce less sGP than EBOV would strengthen the role of sGP in pathogenesis and may explain, in part, why these first two viruses exhibit lower lethality rates than EBOV. Interestingly, viruses belonging to the Marburgvirus genus do not produce sGP but are still able to induce a severe disease that is similar to those of ebolavirus infections (37), suggesting either a nonessential role for sGP in pathogenesis or that marburgviruses possess another mechanism that fulfils the role of sGP. As reviewed previously (17), it was shown in vitro that the less pathogenic 


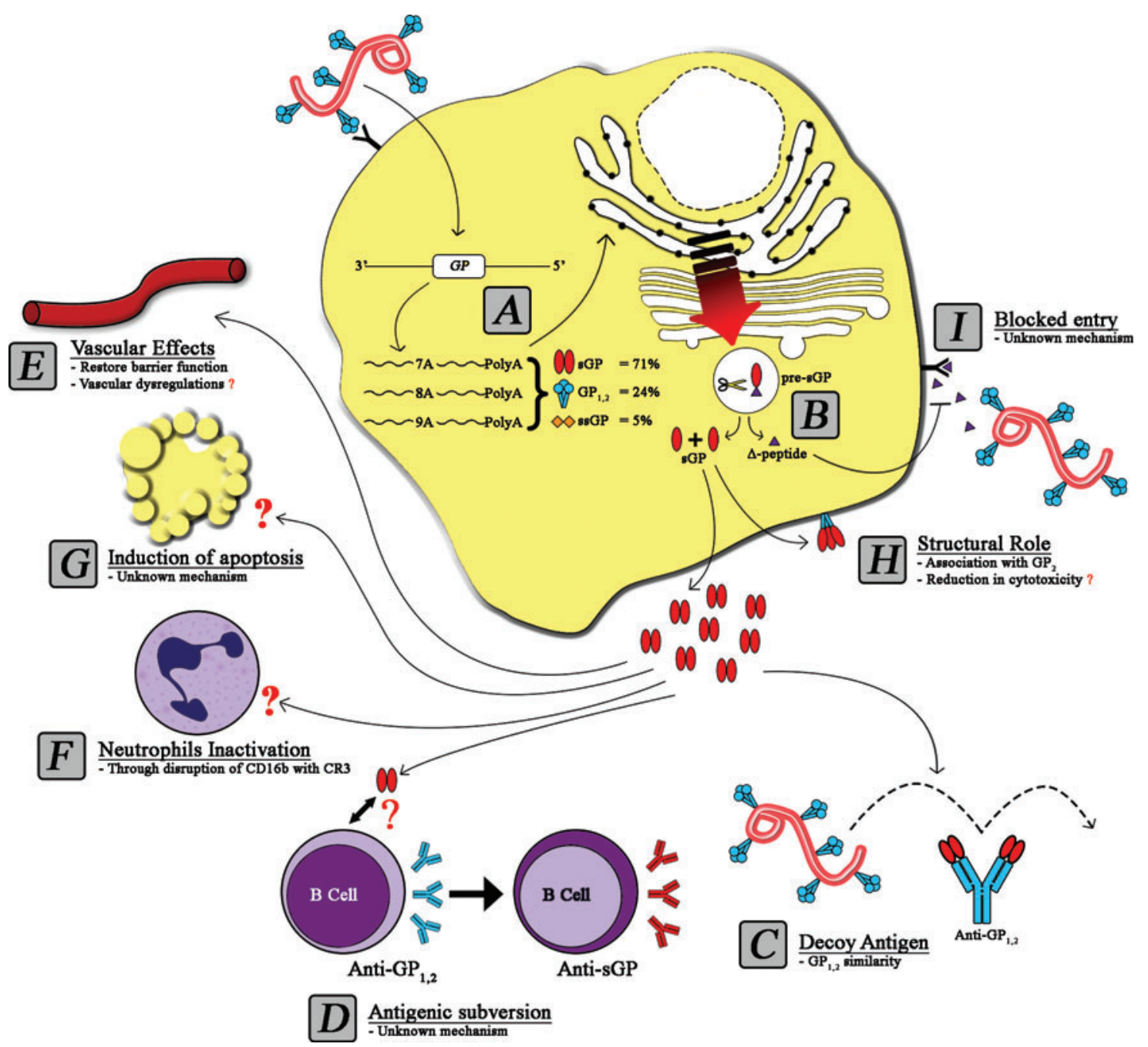

FIG. 1. The impact of secreted glycoprotein (sGP) in Ebola pathogenesis. Following the entry of Ebola into a target cell, the viral genome is released and transcriptional editing of the GP gene leads to the production of three proteins, sGP, GP ${ }_{1,2}$, and ssGP (A). sGP is initially synthesized as pre-sGP, a trans-Golgi-specific precursor that undergoes proteolytic cleavage by cellular proteases, such as furin. This maturation leads to the formation of two monomers, sGP and $\Delta$-peptide (B). sGP monomers then assemble as homodimers in the cytoplasm and are released into the extracellular medium. sGP is currently thought to serve as a decoy antigen $(\mathbf{C})$, be involved in antigenic subversion $(\mathbf{D})$, and play a role in restoring the barrier function of endothelial cells (E). Other proposed effects, such as neutrophil inactivation (F) and induction of apoptosis in bystander cells $(\mathbf{G})$, are less established. sGP monomers were also shown to assemble as heterodimers with $\mathrm{GP}_{2}$, suggesting a structural role for sGP $(\mathbf{H})$; however, the mechanism behind this assembly is still unknown. The $\Delta$-peptide is also secreted from Ebola-infected cells, but at a much slower rate, since it is retained in the cell for a longer period. Once in the extracellular medium, it was shown to be able to block the entry of filoviruses; however, the mechanism is yet to be elucidated (I). Color images available online at www.liebertpub.com/vim

RESTV produces large amounts of sGP (46), whereas the more lethal virus EBOV produces much less by comparison (54). This would argue against a role for sGP in pathogenesis; however, in vivo studies are needed to further investigate this claim. In regard to the conclusion that EBOV is producing small amounts of sGP, although it is the most lethal filovirus, this conclusion was supported by data generated from a guinea pig-adapted variant, making the comparison difficult with human strains (54). Overall, the data generated from future studies on sGP as an important protein to be controlled during EBOV infections may lead to new strategies for vaccine or treatments, taking us one step closer to clinical trials and ultimately control over these viruses.

\section{Acknowledgments}

This work was supported by the Public Health Agency of Canada (PHAC). M.d.L.V. is the recipient of a Master's Research Award from the Canadian Institute for Health Research (CIHR). G.W. is the recipient of a Doctoral Research Award from CIHR.

\section{Author Disclosure Statement}

No competing financial interests exist.

\section{References}

1. Alazard-Dany N, Volchkova V, Reynard O, et al. Ebola virus glycoprotein GP is not cytotoxic when expressed 
constitutively at a moderate level. J Gen Virol 2006;87(Pt 5):1247-1257.

2. Baize S, Leroy EM, Georges AJ, et al. Inflammatory responses in Ebola virus-infected patients. Clin Exp Immunol 2002;128:163-168.

3. Baize S, Leroy EM, Georges-Courbot MC, et al. Defective humoral responses and extensive intravascular apoptosis are associated with fatal outcome in Ebola virus-infected patients. Nat Med 1999;5:423-426.

4. Baize S, Leroy EM, Mavoungou E, and Fisher-Hoch SP. Apoptosis in fatal Ebola infection. Does the virus toll the bell for immune system? Apoptosis 2000;5:5-7.

5. Barrientos LG, Martin AM, Rollin PE, and Sanchez A. Disulfide bond assignment of the Ebola virus secreted glycoprotein SGP. Biochem Biophys Res Commun 2004; 323:696-702.

6. Borchert M, Mutyaba I, Van Kerkhove MD, et al. Ebola haemorrhagic fever outbreak in Masindi District, Uganda: outbreak description and lessons learned. BMC Infect Dis 2011;11:357.

7. Bradfute SB, Braun DR, Shamblin JD, et al. Lymphocyte death in a mouse model of Ebola virus infection. J Infect Dis 2007;196(Suppl 2):S296-S304.

8. Bradfute SB, Swanson PE, Smith MA, et al. Mechanisms and consequences of ebolavirus-induced lymphocyte apoptosis. J Immunol 2010;184:327-335.

9. Chaitanya GV, Steven AJ, and Babu PP. Parp-1 cleavage fragments: signatures of cell-death proteases in neurodegeneration. Cell Commun Signal 2010;8:31.

10. Chan S, Ma M, and Goldsmith M. Differential induction of cellular detachment by envelope glycoproteins of Marburg and Ebola (Zaire) viruses. J Gen Virol 2000;81(Pt 9):21552159.

11. Cole K, Steckbeck J, Rowles J, Desrosiers R, and Montelaro R. Removal of N-linked glycosylation sites in the V1 region of simian immunodeficiency virus gp120 results in redirection of B-cell responses to V3. J Virol 2004;78: $1525-1539$.

12. Cook JD, and Lee JE. The secret life of viral entry glycoproteins: moonlighting in immune evasion. PLoS Pathog 2013;9:1-5.

13. Dowling W, Thompson E, Badger C, et al. Influences of glycosylation on antigenicity, immunogenicity, and protective efficacy of Ebola virus GP DNA vaccines. J Virol 2007;81:1821-1837.

14. Falzarano D, Krokhin O, Van Domselaar G, et al. Ebola sGP - the first viral glycoprotein shown to be C-mannosylated. Virology 2007;368:83-90.

15. Falzarano D, Krokhin O, Wahl-Jensen V, et al. Structurefunction analysis of the soluble glycoprotein, sGP, of Ebola virus. Chembiochem 2006;7:1605-1611.

16. Feldmann H, and Geisbert T. Ebola haemorrhagic fever. Lancet 2011;377:849-862.

17. Feldmann H, Volchkov VE, Volchkova VA, Ströher U, and Klenk HD. Biosynthesis and role of filoviral glycoproteins. J Gen Virol 2001;82(Pt 12):2839-2848.

18. Furmanek A, and Hofsteenge J. Protein C-mannosylation: facts and questions. Acta Biochim Pol 2000;47:781-789.

19. Geisbert TW, Hensley LE, Gibb TR, Steele KE, Jaax NK, and Jahrling PB. Apoptosis induced in vitro and in vivo during infection by Ebola and Marburg viruses. Lab Invest 2000;80:171-186.

20. Geisbert TW, Hensley LE, Larsen T, et al. Pathogenesis of Ebola hemorrhagic fever in cynomolgus macaques: ev- idence that dendritic cells are early and sustained targets of infection. Am J Pathol 2003;163:2347-2370.

21. Gupta M, Spiropoulou C, and Rollin PE. Ebola virus infection of human PBMCs causes massive death of macrophages, CD4 and CD8 T cell sub-populations in vitro. Virology 2007;364:45-54.

22. Hausmann S, Garcin D, Delenda C, and Kolakofsky D. The versatility of paramyxovirus RNA polymerase stuttering. J Virol 1999;73:5568-5576.

23. Hensley LE, Young HA, Jahrling PB, and Geisbert TW. Proinflammatory response during Ebola virus infection of primate models: possible involvement of the tumor necrosis factor receptor superfamily. Immunol Lett 2002;80:169-179.

24. Ito $\mathrm{H}$, Watanabe $\mathrm{S}$, Takada $\mathrm{A}$, and Kawaoka $\mathrm{Y}$. Ebola virus glycoprotein: proteolytic processing, acylation, cell tropism, and detection of neutralizing antibodies. J Virol 2001;75:1576-1580.

25. Iwasa A, Shimojima M, and Kawaoka Y. sGP serves as a structural protein in Ebola virus infection. J Infect Dis 2011;204 Suppl (Suppl 3):S897-S903.

26. Kang S-M, Quan FS, Huang C, et al. Modified HIV envelope proteins with enhanced binding to neutralizing monoclonal antibodies. Virology 2005;331:20-32.

27. Kindzelskii AL, Yang Z, Nabel GJ, Todd RF, and Petty HR. Ebola virus secretory glycoprotein (sGP) diminishes Fc gamma RIIIB-to-CR3 proximity on neutrophils. J Immunol 2000;164:953-958.

28. Kolakofsky D, Roux L, Garcin D, and Ruigrok RWH. Paramyxovirus mRNA editing, the "rule of six" and error catastrophe: a hypothesis. J Gen Virol 2005;86(Pt 7):18691877.

29. Kuhn JH, Bào Y, Bavari S, et al. 2013. Virus nomenclature below the species level: a standardized nomenclature for filovirus strains and variants rescued from cDNA. Arch Virol 2014;159:1229-1237.

30. Kulkarni S, Volchkova V, Basler CF, Palese P, Volchkov VE, and Shaw ML. Nipah virus edits its $\mathrm{P}$ gene at high frequency to express the V and W proteins. J Virol 2009; 83:3982-3987.

31. Lee JE, Fusco ML, Hessell AJ, Oswald WB, and Burton DR. Structure of the Ebola virus glycoprotein bound to a human survivor antibody. Nature 2009;454:177-182.

32. Maruyama J, Miyamoto H, Kajihara M, et al. Characterization of the envelope glycoprotein of a novel filovirus, lloviu virus. J Virol 2014;88:99-109.

33. Maruyama T. Ebola virus, neutrophils, and antibody specificity. Science 1998;282:843.

34. Maruyama T, Parren PW, Sanchez A, et al. Recombinant human monoclonal antibodies to Ebola virus. J Infect Dis 1999;179:S235-S239.

35. Marzi A, Engelmann F, Feldmann F, et al. Antibodies are necessary for rVSV/ZEBOV-GP-mediated protection against lethal Ebola virus challenge in nonhuman primates. Proc Natl Acad Sci U S A 2013;110:1893-1898.

36. Mehedi M, Falzarano D, Seebach J, et al. A new Ebola virus nonstructural glycoprotein expressed through RNA editing. J Virol 2011;85:5406-5414.

37. Mehedi M, Hoenen T, Robertson S, et al. Ebola virus RNA editing depends on the primary editing site sequence and an upstream secondary structure. PLoS Pathog 2013;9: e1003677.

38. Miller EH, Obernosterer G, Raaben M, et al. Ebola virus entry requires the host-programmed recognition of an intracellular receptor. EMBO J 2012;31:1947-1960. 
39. Mohan GS, Li W, Ye L, Compans RW, and Yang C. Antigenic subversion: a novel mechanism of host immune evasion by Ebola virus. PLoS Pathog 2012;8:e1003065.

40. Mori K, Sugimoto C, and Ohgimoto S. Influence of glycosylation on the efficacy of an Env-based vaccine against simian immunodeficiency virus SIVmac239 in a macaque AIDS model. J Virol 2005;79:10386-10396.

41. Negredo A, Palacios G, Vázquez-Morón S, et al. Discovery of an ebolavirus-like filovirus in Europe. PLoS Pathog 2011;7:e1002304.

42. Olejnik J, Alonso J, Schmidt KM, et al. Ebola virus does not block apoptotic signaling pathways. J Virol 2013;87: 5384-5396.

43. Peters CJ, Sanchez A, Feldmann H, Rollin PE, Nichol ST, and Ksiazek TG. Filoviruses as emerging pathogens. Virology 1994;5:147-154.

44. Radoshitzky SR, Warfield KL, Chi X, et al. Ebolavirus delta-peptide immunoadhesins inhibit marburgvirus and ebolavirus cell entry. J Virol 2011;85:8502-8513.

45. Reitter J, Means R, and Desrosiers R. A role for carbohydrates in immune evasion in aids. Nat Med 1998;4:679684.

46. Sanchez A, Trappier SG, Mahy BW, Peters CJ, and Nichol ST. The virion glycoproteins of Ebola viruses are encoded in two reading frames and are expressed through transcriptional editing. Proc Natl Acad Sci U S A 1996;93: 3602-3607.

47. Sanchez A, Yang ZY, Xu L, Nabel GJ, Crews T, and Peters CJ. Biochemical analysis of the secreted and virion glycoproteins of Ebola virus. J Virol 1998;72:6442-6447.

48. Sui J, and Marasco WA. Evidence against Ebola virus sGP binding to human neutrophils by a specific receptor. Virology 2002;303:9-14.

49. Vanderzanden L, Bray M, Fuller D, et al. DNA vaccines expressing either the GP or NP genes of Ebola virus protect mice from lethal challenge. Virology 1998;246: 134-144.

50. Volchkova VA, Dolnik O, Martinez MJ, Reynard O, and Volchkov VE. Genomic RNA editing and its impact on Ebola virus adaptation during serial passages in cell culture and infection of guinea pigs. J Infect Dis 2011;204(Suppl 3):S941-S946.

51. Volchkova VA, Feldmann H, Klenk HD, and Volchkov VE. The nonstructural small glycoprotein sGP of Ebola virus is secreted as an antiparallel-orientated homodimer. Virology 1998;250:408-414.

52. Volchkova VA, Klenk HD, and Volchkov VE. Deltapeptide is the carboxy-terminal cleavage fragment of the nonstructural small glycoprotein sGP of Ebola virus. Virology 1999;265:164-171.

53. Volchkov VE, Becker S, Volchkova VA, et al. GP mRNA of Ebola virus is edited by the Ebola virus polymerase and by T7 and vaccinia virus polymerases. Virology 1995;214: 421-430.
54. Volchkov VE, Chepurnov AA, Volchkova VA, Ternovoj VA, and Klenk HD. Molecular characterization of guinea pig-adapted variants of Ebola virus. Virology 2000;277: 147-155.

55. Volchkov VE, Feldmann H, Volchkova VA, and Klenk HD. Processing of the Ebola virus glycoprotein by the proprotein convertase furin. Proc Natl Acad Sci U S A 1998;95:5762-5767.

56. Volchkov VE, Volchkova VA, Muhlberger E, et al. Recovery of infectious Ebola virus from complementary DNA: RNA editing of the GP gene and viral cytotoxicity. Science 2001;291:1965-1969.

57. Wahl-Jensen V, and Afanasieva T. Effects of Ebola virus glycoproteins on endothelial cell activation and barrier function. J Virol 2005;79:10442-10450.

58. Wauquier N, Becquart P, Padilla C, Baize S, and Leroy EM. Human fatal zaire Ebola virus infection is associated with an aberrant innate immunity and with massive lymphocyte apoptosis. PLoS Negl Trop Dis 2010;4:e837.

59. Wilson JA, Hevey M, Bakken R, et al. Epitopes involved in antibody-mediated protection from Ebola virus. Science 2000;287:1664-1666.

60. Wolf K, Beimforde N, Falzarano D, Feldmann H, and Schnittler H-J. The Ebola virus soluble glycoprotein (sGP) does not affect lymphocyte apoptosis and adhesion to activated endothelium. J Infect Dis 2011;204(Suppl 3):S947S952.

61. Wong G, Kobinger GP, and Qiu X. Characterization of host immune responses in Ebola virus infections. Expert Rev Clin Immunol 2014;10:1-10.

62. Wong G, Richardson JS, Pillet S, et al. Immune parameters correlate with protection against Ebola virus infection in rodents and nonhuman primates. Sci Transl Med 2012;4: 158 ra146.

63. Xu L, Sanchez A, Yang Z, and Zaki S. Immunization for Ebola virus infection. Nat Med 1998;4:37-42.

64. Yang Z, Delgado R, Xu L, et al. Distinct cellular interactions of secreted and transmembrane Ebola virus glycoproteins. Science 1998;279:1034-1037.

65. Yang ZY, Duckers HJ, Sullivan NJ, Sanchez A, Nabel EG, and Nabel GJ. Identification of the Ebola virus glycoprotein as the main viral determinant of vascular cell cytotoxicity and injury. Nat Med 2000;6:886-889.

Address correspondence to:

Dr. Xiangguo Qiu

Special Pathogens Program

National Microbiology Laboratory

Public Health Agency of Canada 1015 Arlington Street

Winnipeg, Manitoba R3E $3 R 2$

Canada

E-mail: xiangguo.qiu@phac-aspc.gc.ca 